\title{
Heating Control Design of a Space Camera
}

\author{
Qing-Lei Zhao ${ }^{1,2,{ }^{*}}$, Ke-Hui Long ${ }^{1}$, Feng-Wei Guan ${ }^{1}$ and Liang Huang ${ }^{1}$ \\ ${ }^{1}$ Changchun Institute of Optics, Fine Mechanics and Physics, Chinese Academy of \\ Sciences, Changchun 130033, China \\ ${ }^{2}$ University of Chinese Academy of Sciences, Beijing 100039, China \\ coldsun@sina.com,lkh-email@sohu.com,buaaguan@sohu.com, \\ hezqxfk9@126.com
}

\begin{abstract}
When a space camera works on the track, it presents in vacuum environment, under the condition of low temperatures, the camera can only maintain its temperature level by absorption of radiation. If stayed in this environment for a long time, mechanical structure of the space camera may become brittle, lose strength, and an exfoliation phenomenon may appear, in addition, abnormal operation and malfunction of electronic equipments may also occur, these may affect imaging quality of the camera. In this paper, a heating control method is designed for a space flight off-axis camera. Firstly, heating control working platform of the camera is introduced, a design method of heating control is determined. After that, heating control treatment procedure is introduced. Finally, the ground temperature monitor is used to validate correctness of the method designed. The comparison diagram before and after thermal analysis and temperature data obtained from experiments are presented, results show that, the heating control method is simple and effective, it has wide applicability and strong generalization performance.
\end{abstract}

Keywords: space camera, thermal control system, remote sensing, thermal analysis

\section{Introduction}

Temperature is an important environment condition. It may affect occurrence and development of each thing on the earth. The main work environment of a space camera is the cosmic space beyond the earth's atmosphere. The thermal environment there existed is totally different from that on the earth $[1,2]$. In order to make the camera operated in the scheduled environment, temperature variation of each point of the camera should be properly arranged and controlled timely $[3,4]$.

Low-temperature environment and uneven temperature distribution may have pessimistic effects on electronic equipments, optical modules, automatic equipments and so on [5-9]. These negative effects include generation of thermal stress and deformation, fatigue damage, mechanical breakage or permanent deformation of electron devices, so these may significantly affect working performance of the whole system.

Currently, most of the thermic control studies about space cameras mainly focus on thermo analysis, high effective cooling and heat dissipation [10-15], heat-resistant surface coating materials and so on; there is no deep research on thermal control devices and strategies. At present, electric heating thermostats are usually used for heating control of space cameras. So the thermal control strategies are very important [16-19].

This study starts from engineering practices, based on characteristic analysis of a camera subsystem on a satellite platform. Requirements of temperature uniformity and stability for the camera are considered, a heating control method which based on SMJ320C30 controller is proposed, in the method, there are 80 heating channels.

* Corresponding Author 
Temperature of channels can be controlled independently, there are 160 collection channels. Two collection channels are distributed for each heating point, which can improve equipment stability. Treatment procedures of temperature collection and heating control can be obtained. Validity and stability of the temperature control method can also be verified.

\section{Hardware System}

Heating controller is used for temperature monitoring and control of the space camera. Its main functions include 1553B communication (reception of target temperature, collection of strategy information, sending of real time temperature data), temperature control of camera, temperature collection. Composition diagram of the system is shown in Figure 1 .

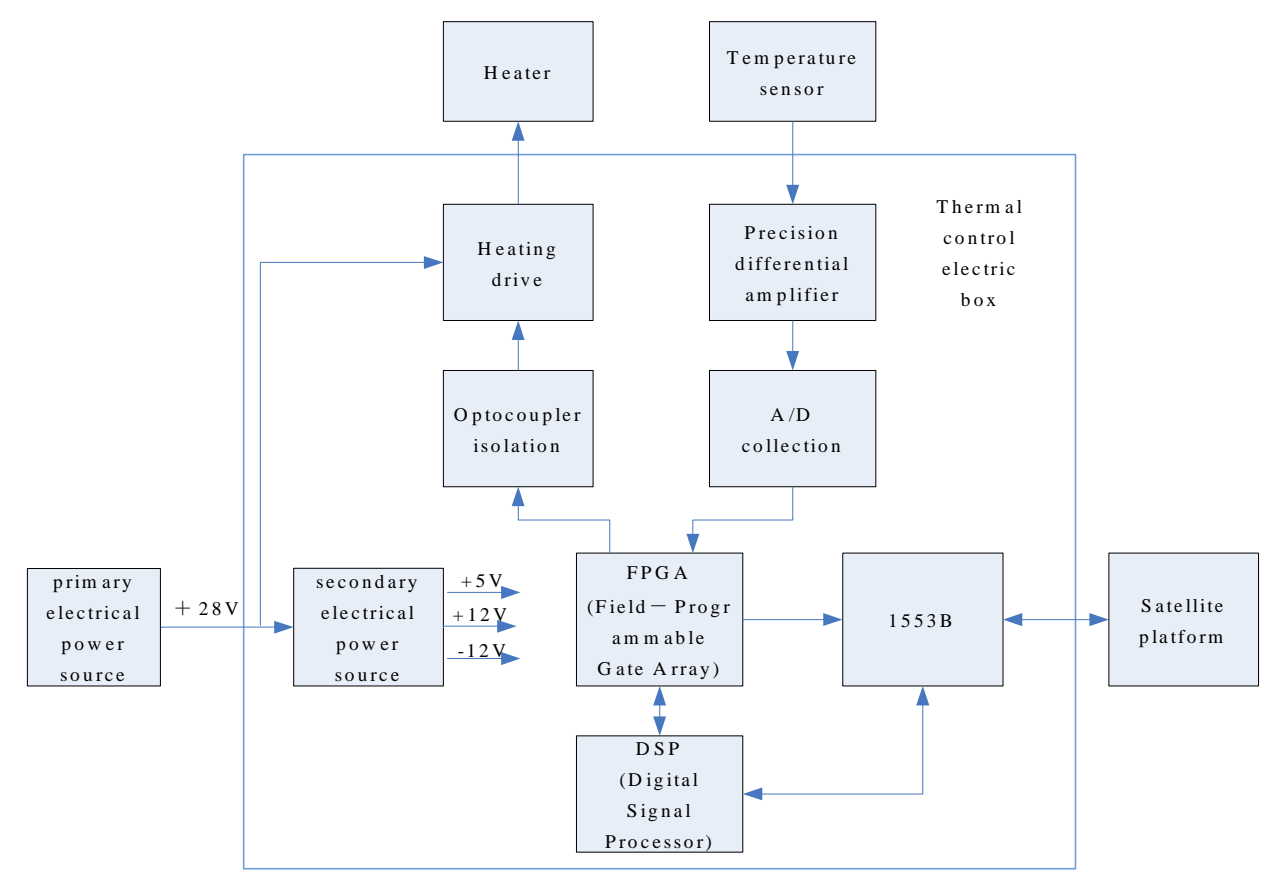

Figure 1. Heating Control System Structure Diagram

Heating controller procedure is solidified in FLASH (Flash Memory), after power up, the procedure runs, it has high reliability.

RAM (Random-Access Memory) is a data-storage area. It can be used for preservation of variables, stacks and related parameter tabulations, including reference resistance, thermal resistance, and reference voltage of each heating channel, which required for various temperature calculations in the operational process of heating control program, in order to improve calculation accuracy. Address section is $80000 \mathrm{H}$ - $\mathrm{FFFFH}$.

Heating controller communicates with the satellite platform by $1553 \mathrm{~B}$ bus. It can interact with FPGA by address visiting.

\section{Software System}

\subsection{Interaction between Satellite Platform and Heating Controller}

Based on resources provided by satellite platform, information and sub addresses, which received by heating controller through 1553B is presented in Table 1. 
Table 1. Content and Sub-address of Received Message Data

\begin{tabular}{|c|c|}
\hline sub-address & Content \\
\hline SA1 & $\begin{array}{c}\text { Collection type and target temperature of channel } \\
\text { 1-channel 32(32 words) }\end{array}$ \\
\hline SA2 & $\begin{array}{c}\text { Collection type and target temperature of channel } \\
\text { 33-channel 64(32 words) }\end{array}$ \\
\hline SA3 & $\begin{array}{c}\text { Collection type and target temperature of channel } \\
\text { 65-channel 80(16 words) }\end{array}$ \\
\hline
\end{tabular}

The ground control platform sends collection type and target temperature to the satellite platform (a 5K RAM region in the satellite platform is used for preservation of orders and parameters from the ground). The satellite can send the information to the heating controller through 1553B protocol, sub-address $1 \sim 3$ are used for reception of channel collection type and target temperature of 80 channels from the platform. The format is shown in Table 2 .

\section{Table 2. Collection Type Code and the Target Temperature Format of} Each Channel

\begin{tabular}{|c|c|}
\hline Bit & Content \\
\hline Bit0 Bit9 & The target temperature code \\
\hline Bit10 Bit12 & 0 \\
\hline Bit13 & Heating control code \\
\hline Bit14 Bit15 & Collection type code \\
\hline
\end{tabular}

Collection type code, the format is shown in Table 3.

Table 3. Collection Type Code Format

\begin{tabular}{|c|c|c|}
\hline Bit15 & Bit14 & Content \\
\hline 0 & 0 & No collecting \\
\hline 0 & 1 & Collection of channel A \\
\hline 1 & 0 & Collection of channel B \\
\hline 1 & 1 & Collection of $(\mathrm{A}+\mathrm{B}) / 2$ \\
\hline
\end{tabular}

Heating control code, the format is shown in Table 4.

Table 4. Heating Control Code Format

\begin{tabular}{|c|c|}
\hline Bit13 & Content \\
\hline 0 & No heating \\
\hline 1 & Heating \\
\hline
\end{tabular}

Data range of target temperature code is 0 -800(it is obtained by practical temperature $\times 10$ ). It occupies 10 bits by binary format (bit0 bit9). 
Heating controller sends collected temperature and heating status to the satellite platform by 1553B serial bus communication. These are downloaded to the ground control center for temperature monitoring, information and sub addresses are shown in Table 5.

Table 5. Content and Sub-address of Sent Message Data

\begin{tabular}{|c|c|}
\hline sub-address & Content \\
\hline SA1 & $\begin{array}{c}\text { Heating status and current temperature of channel } \\
\text { 1-channel32(32 words) }\end{array}$ \\
\hline SA2 & $\begin{array}{c}\text { Heating status and current temperature of channel } \\
\text { 33-channel64(32 words) }\end{array}$ \\
\hline SA3 & $\begin{array}{c}\text { Heating status and current temperature of channel } \\
\text { 65-channel80(16 words) }\end{array}$ \\
\hline
\end{tabular}

Heating status and temperature format of each channel is shown in Table 6.

Table 6. Heating Status and Current Temperature Format of Each Channel

\begin{tabular}{|c|c|}
\hline Bit & Content \\
\hline Bit0 Bit11 & The current temperature code \\
\hline Bit12 Bit13 & The heating status code \\
\hline Bit14 Bit15 & 0 \\
\hline
\end{tabular}

Heating status code, the format is shown in Table 7.

Table 7. Heating Status Code Format

\begin{tabular}{|c|c|c|}
\hline Bit13 & Bit12 & Content \\
\hline 0 & 0 & No heating \\
\hline 0 & 1 & $30 \%$ Pulse heating \\
\hline 1 & 0 & $60 \%$ Pulse heating \\
\hline 1 & 1 & Continuous heating \\
\hline
\end{tabular}

The current temperature code has 12 digitals. Normal temperature range is $0-800$. It occupies 10 bits by binary format (bit0 bit9), when the temperature is out of range of $0-800$, it can be taken as an error value. The code value is actual temperature $\times 10$, practical operating ambient temperature of the camera is$30^{\circ} \mathrm{C} \sim 50^{\circ} \mathrm{C}$, the temperature value should be divided by 10 and minus 30 for the ground monitoring equipments. Correct temperature is shown on display after calculated. Temperature precision after calculation is $0.1{ }^{\circ} \mathrm{C}$. When temperature collection is stopped, the current temperature code is set as 0xfff and sent to the ground detection equipments, so it can be distinguished from other temperature data. 


\subsection{Interaction of Heating Controller DSP and FPGA}

Interaction of heating controller DSP and FPGA can be performed by address bus, addresses used and their meanings are shown in Table 8 .

Table 8. Addresses Used and Meanings

\begin{tabular}{|c|c|}
\hline Address & Content \\
\hline 0x60001 & Selection of step1 analogue switch channel \\
\hline 0x60002 & Selection of step2 analogue switch channel \\
\hline 0x60003 & Control of AD (Analog to Digital) change-over switch \\
\hline 0x60004 & Temperature read \\
\hline 0x70000 & Heating control \\
\hline
\end{tabular}

Analogue switch is used for selection of 160 collection channels. 160 channels are divided into two separate collection system. Group A and group B, there are 80 channels in each group. Where, there are 10 channels in step1 analogue switch and 16 channels in step 2 analogue switch. They are connected by the series system, so the controller can control temperature collection of 160 channels $(10 \times 16)$.

DSP writes FH to control address of the AD change-over switch, then FPGA works for AD convert. INT2 interrupt appears after converting, DSP reads temperature from addresses in interrupt processing, temperature corresponding code is obtained after $\mathrm{AD}$ converted, practical operating ambient temperature is obtain ed by follow-up calculation.

DSP can independently perform heating control according to temperature collected from different channels, heating control format is shown in Table 9.

Table 9. Heating Control Instruction Format

\begin{tabular}{|c|c|}
\hline Bit & Content \\
\hline Bit0 Bit7 & Heating mode \\
\hline Bit8 Bit15 & Heating channel \\
\hline
\end{tabular}

Heating channel range is 1 to 80 , representing the 80 -way heating channels, data range of heating types is $0 \sim 100,0$ refers to no heating, 100 refers to continuous heating, if $30 \%$ pulse heating is performed, set the value as 30 , if $60 \%$ pulse heating is performed, set the value as 60 .

\section{Heating Control Method}

Heating control method mainly includes temperature collection and heating set. Collection channels are distributed according to form and position of heater bands. Automatic heating set can be performed by temperature collection, temperature calculation and temperature comparison of each channel.

\subsection{Temperature Collection}

DSP uses 1 out of 16 selectors (MAXIM company, model MAX306CPI) as analogue switch, and can select collection channels through controlling analogue switch. 11 slices of analogue switches (two steps) are required for 160channels. The circuit design schematic diagram is shown in Figure 2. 


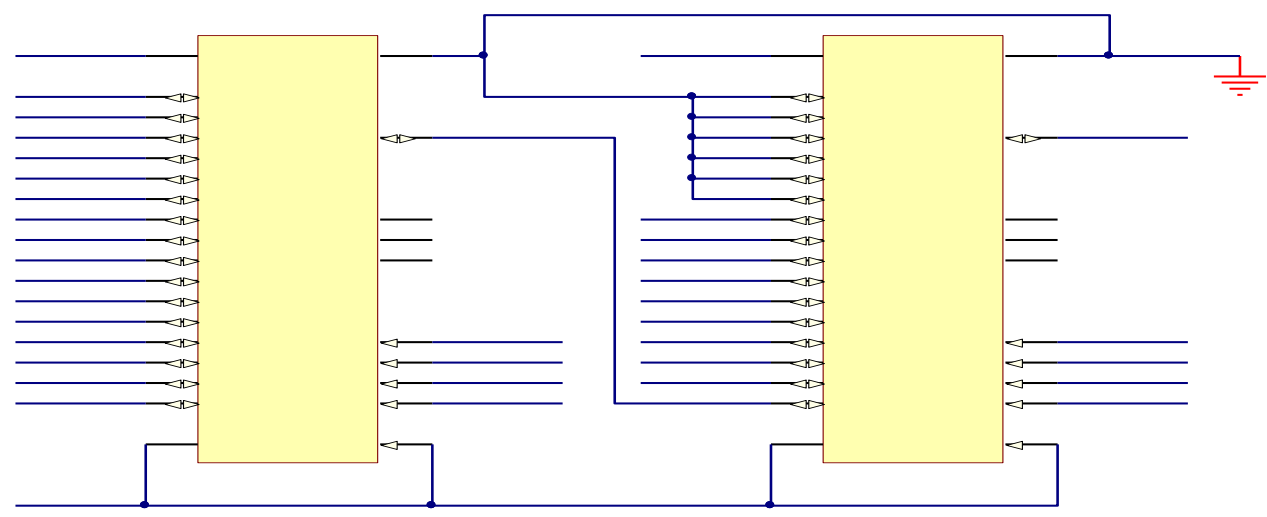

Figure 2. Collection Channel Switching Circuit Schematic

Using bridge circuit, it can transform resistance value to voltage. An op amp amplifier can magnify voltage, then the analog-digital converter can transform to digital quantity and output to DSP for processing, the circuit design schematic diagram is shown in Figure 3.

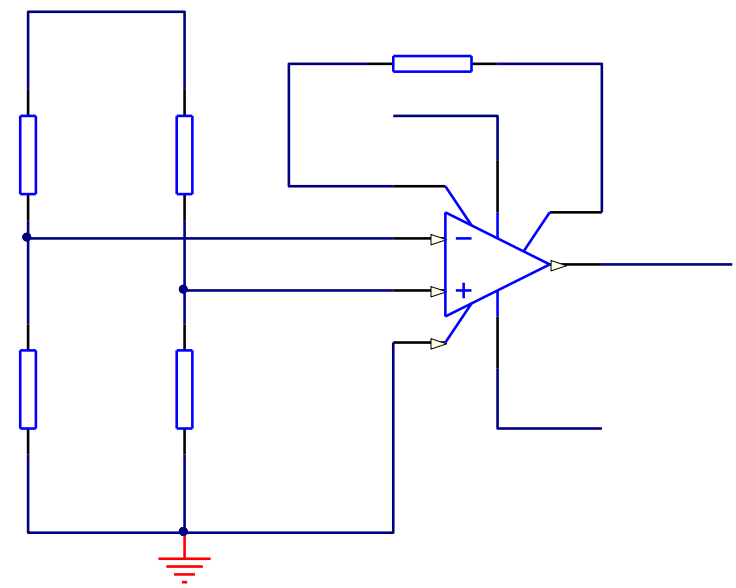

Figure 3. Bridge Circuit Schematic

AD584 drives Wheatstone bridge in the diagram. Using AD620 as op amp amplifier, service voltage is $\pm 15 \mathrm{~V}$. Reference voltage is input into the negative terminal of the op amp amplifier, and voltage to be tested is input into the plus end. It adopts AD574AL as ADC (Analog to Digital Converter), service voltage is $\pm 15 \mathrm{~V}$, measuring range is $\pm 10 \mathrm{~V}$, and the maximum linear error is $\pm 1 / 2 \mathrm{LSB}$. In order to obtain the maximum sampling precision, reasonable op amp gain is selected, suitable bridge arm is designed, and output of the op amp amplifier should be mostly close to measuring range of the analog-digital converter. So it is obtained that

$$
\begin{gathered}
\left(V_{+\max }-V_{-}\right) \times G=+10 \\
\left(V_{+ \text {min }}-V_{-}\right) \times G=-10
\end{gathered}
$$

Where, $V_{+\max } / V_{+\min }$ is the maximum/minimum positive voltage, $V$ - is the negative voltage. $G$ is the gain of the op amp amplifier, Substituting Equation (1) into Equation (2) gives 


$$
V_{+\max }-V_{+\min }=\frac{20}{G}
$$

Figure 3 shows that:

$$
\begin{gathered}
V_{+}=V_{R K R E F} \times \frac{R_{t}}{R_{2}+R_{t}} \\
V_{-}=V_{R K R E F} \times \frac{R_{3}}{R_{1}+R_{3}}
\end{gathered}
$$

Where, $V_{R K R E F}$ is the reference voltage, set $V_{R K R E F}=+10 \mathrm{~V} ; R_{1}, R_{2}, R_{3}$ is the selected resistance value of circuit; $R_{t}$ is the thermistor that can get from the temperatureresistance curve. When the temperature $\mathrm{t}$ is $-30^{\circ} \mathrm{C}, R_{t}=100.7679 \mathrm{~K} \Omega$; when $\mathrm{t}$ is $+50^{\circ} \mathrm{C}, R_{t}=1.7604 \mathrm{~K} \Omega$; when $\mathrm{t} \in\left(-30^{\circ} \mathrm{C},+50^{\circ} \mathrm{C}\right), 100.7679 \mathrm{~K} \Omega \leq R_{t} \leq 1.7604 \mathrm{~K} \Omega$. From Equation (4), $V_{+\max }$ and $V_{+\min }$ can be obtained as

$$
\begin{aligned}
& V_{+ \text {max }}=10 \times \frac{100.7679}{R_{2}+100.7679} \\
& V_{+ \text {m in }}=10 \times \frac{1.7604}{R_{2}+1.7604}
\end{aligned}
$$

From Equation (3), it can be calculated as

$R_{2}{ }^{2}+(102.5283-49.5038 G) R_{2}+176.04=0$

Take $G=4, R_{2}=93$ or $1.8 \mathrm{~K} \Omega$, in order to reduce the power consumption of the bridge circuit, $R_{2}$ can be selected as $100 \mathrm{~K} \Omega$, from Equations (6)-(7), it can be obtained as

$$
\left\{\begin{array}{l}
V+\text { max } \\
+5.019 \mathrm{~V} \\
V_{+ \text {min }}=+0.173 \mathrm{~V}
\end{array}\right.
$$

$V_{+\max }$ and $V_{+\min }$ are substituted into Equations (1)-(2), then it is known that $V$ $\in(+2.519 \mathrm{~V},+2.673 \mathrm{~V})$. Take $R_{l}=100 \mathrm{~K} \Omega$, from Equation (5), it can be obtained that $R_{3} \in(33.67 \mathrm{~K} \Omega, 36.48 \mathrm{~K} \Omega)$. Take $R_{3}=34.8 \mathrm{~K} \Omega, V-=+2.582 \mathrm{~V}$, so main parameters of the bridge circuit are as follows:

Resistance value of bridge arm: $R_{1}=100 \mathrm{~K} \Omega, R_{2}=100 \mathrm{~K} \Omega, R_{3}=34.8 \mathrm{~K} \Omega$;

Output voltage: $V_{-}=+2.582 \mathrm{~V}, V_{+} \in(+0.173 \mathrm{~V},+5.019 \mathrm{~V})$;

Voltage difference: $V_{+}-V_{-} \in(-2.409 \mathrm{~V},+2.437 \mathrm{~V})$;

Minimum operating resistance:

$$
1\left(\frac{1}{100+34.8}+\frac{1}{100+1.7604}\right)=57.99 \mathrm{k} \Omega \text {; }
$$

Maximum operating current: $10 \mathrm{~V} / 57.99 \mathrm{~K} \Omega=0.172 \mathrm{~mA}$.

An external gains set resistance $R_{G}$ can adjust gain $G$ of operational amplifier AD620. They are designed as

$$
\begin{aligned}
& G=\frac{49.4 \mathrm{~K} \Omega}{R_{G}}+1 \\
& R_{G}=\frac{49.4 \mathrm{k} \Omega}{G-1}
\end{aligned}
$$

Let $G=4$, from Equation (10), it is obtained that $R_{G}=16.4667 \mathrm{~K} \Omega$; let $R_{G}=16.5 \mathrm{~K} \Omega$, from Equation (9), it is obtained that $G=3.994$. Output of the operational amplifier $V_{o}=G \times\left(V_{+}-V_{-}\right)$, it is given that $V_{o} \in(-9.621 \mathrm{~V},+9.733 \mathrm{~V})$. AD574 can detect analog input of $\pm 10 \mathrm{~V}$, so $V_{o}$ is within the legal range.

Thermistor is selected from series MF61 of NTC. Before used, it performs screening, thermo sensitive calibration tests, and formula fitting for data obtained from the tests, to improve measuring veracity. So it is designed as 
$T=2 C \int_{\left(-B+\sqrt{B^{2}-4 C(A-\operatorname{Ln} R)}\right)}-273.15$

where, $T$ is the calculated result of temperature in ${ }^{\circ} \mathrm{C} ; R$ is thermistor resistance value in $\Omega ; A, B, C$ is the thermistor coefficient, which can be obtained from formula fitting. DSP selects coefficient of each thermistor through looking up table, and uses them to calculate according to the formula.

\subsection{Heating Devices}

After temperature collection, DSP compares and judges target temperatures received, and it can perform heating set automatically, its processing flow chart is shown as follows.

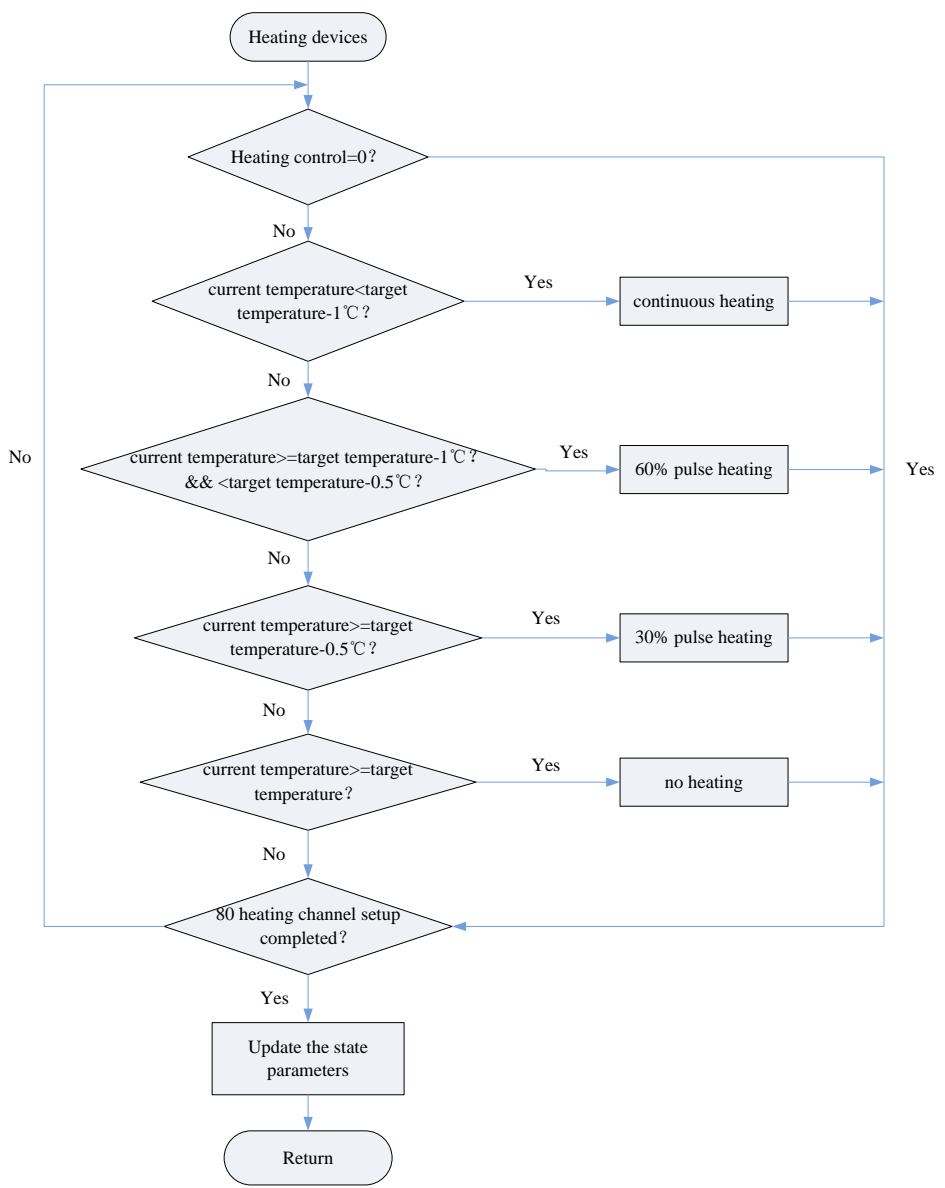

Figure 2. Heating Devices of Flow Chart

\section{Test and Analysis of Experiments}

\subsection{Thermal Analysis Before the Experiment}

A space flight camera performs a thermo analysis, temperature distribution before and after the analysis is shown in Figure 5. The figure shows that, after analysis, heat distribution consistency of the camera is better, so it can confirm operation temperature of each heating point. 

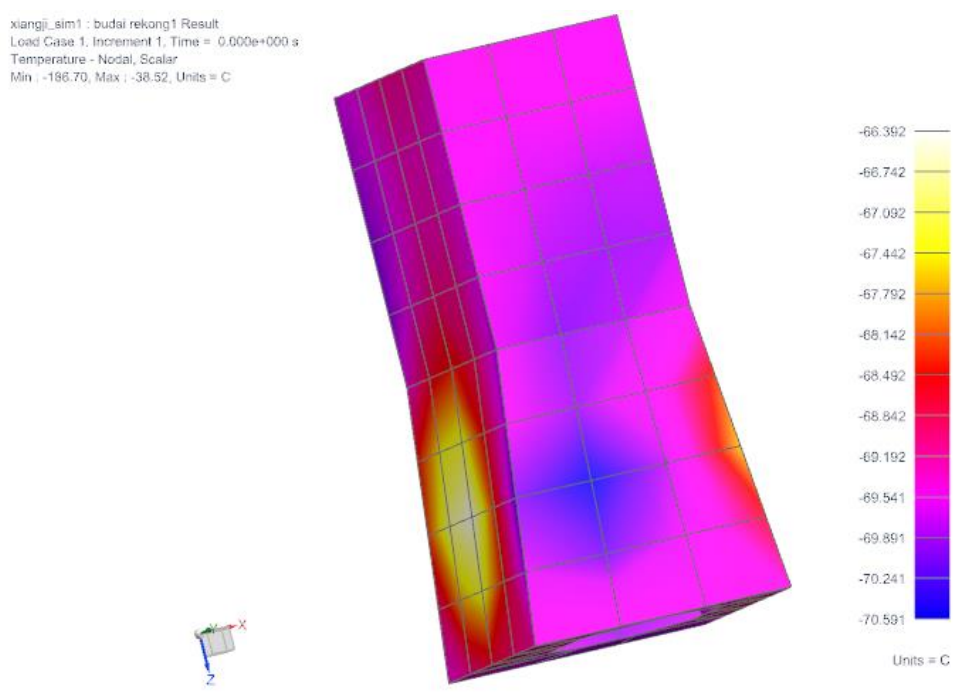

(a) Cloud Picture of Temperature Distribution before Heating Control
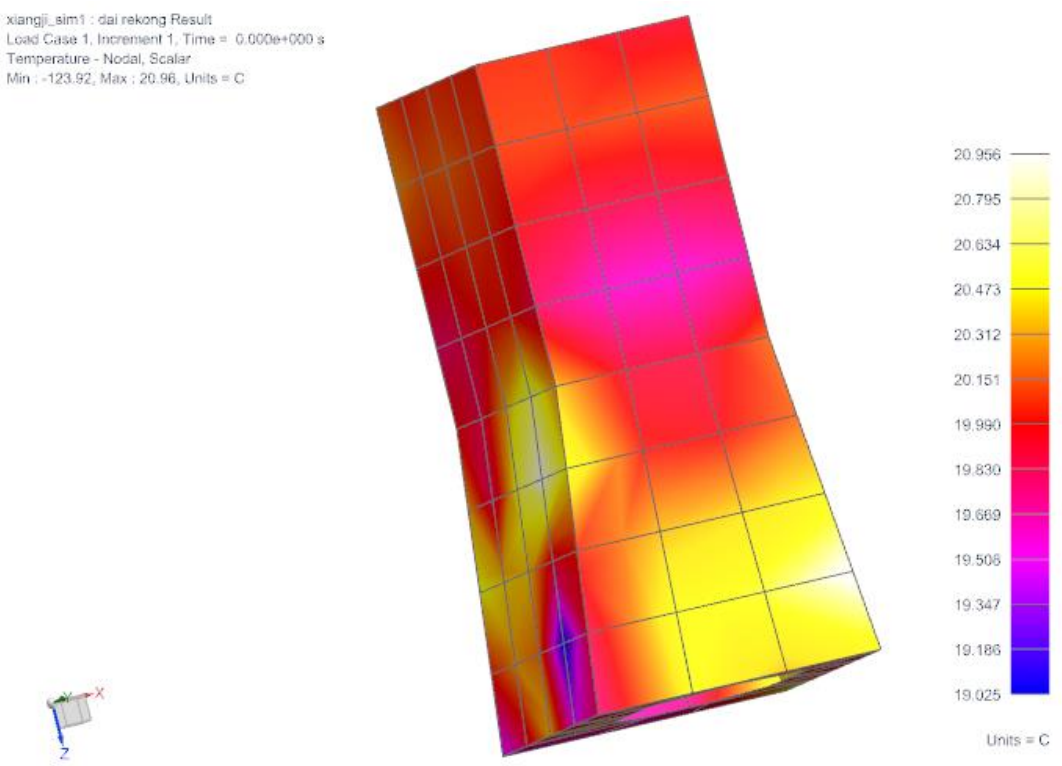

(b) Cloud Picture of Temperature Distribution after Heating Control

\section{Figure 3. Comparison Chart of Temperature Distribution Before and After Heating Control}

\subsection{Heating Control Stability Test}

Proceed from actual engineering, it sets up target temperature of each heating channel according to results after thermo analysis, its setup interface is shown in Figure 6, the heating controller performs heating control, set state return interface is shown in Figure 7. The interface shows that, the practical controlled temperature is accord with the target temperature. 


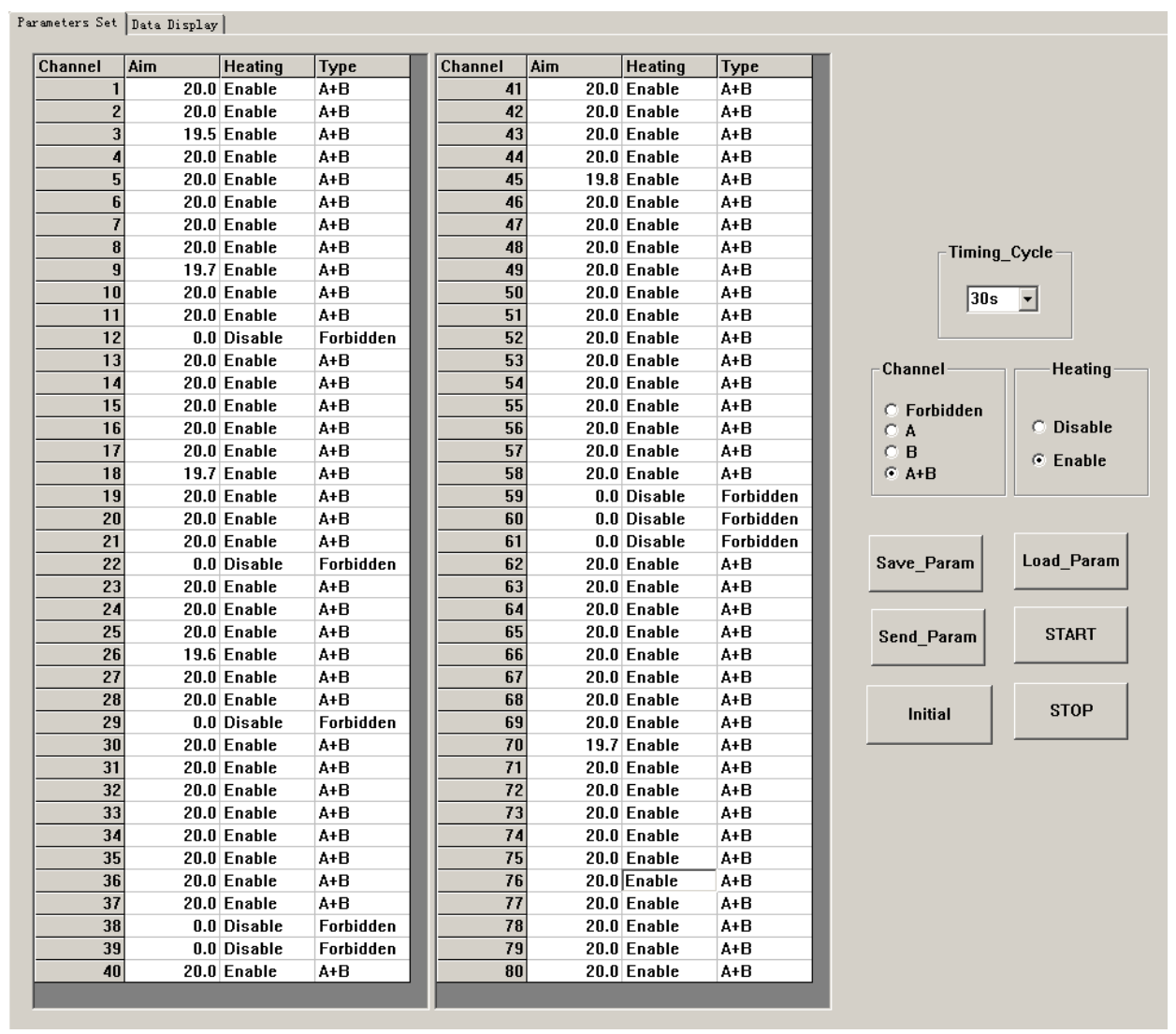

Figure 6. Setup Interface of Heating Control Parameters 


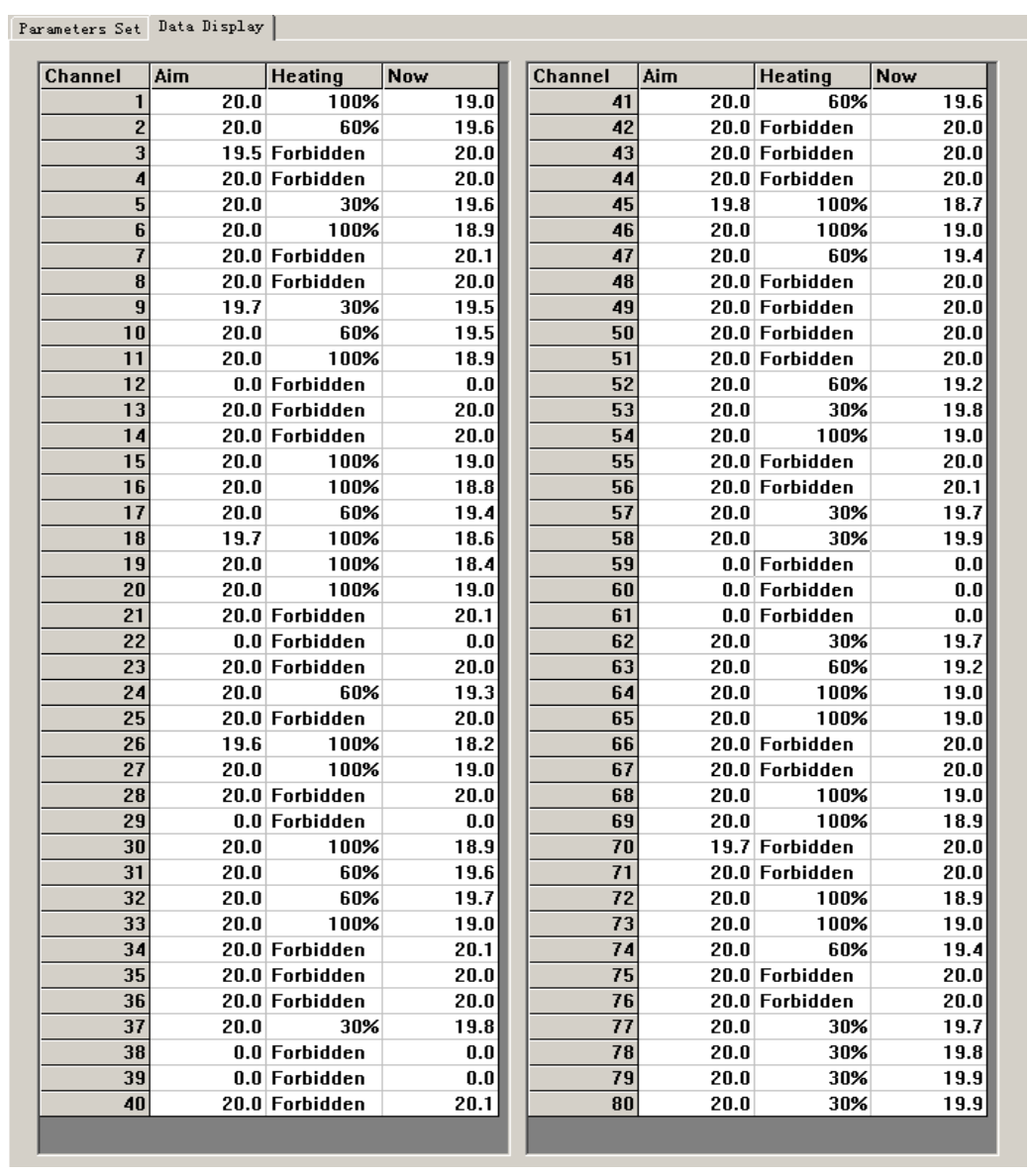

Figure 7. Display Interface of Temperature Collection

\subsection{Heating Control Validity Test}

This is a 96 hours' low temperature $\left(-40^{\circ} \mathrm{C}\right)$ test in a vacuum, heating controller returns state parameter within a cycle of $30 \mathrm{~s}$, we analyze the partial parameter result of a heating channel, which is compared with target temperature. Its corresponding curve is shown in Figure 8 . The figure shows that, the temperature difference of practical temperature and target temperature is within the range of $-0.5^{\circ} \mathrm{C} \sim+0.2^{\circ} \mathrm{C}$, heating control accuracy is high.

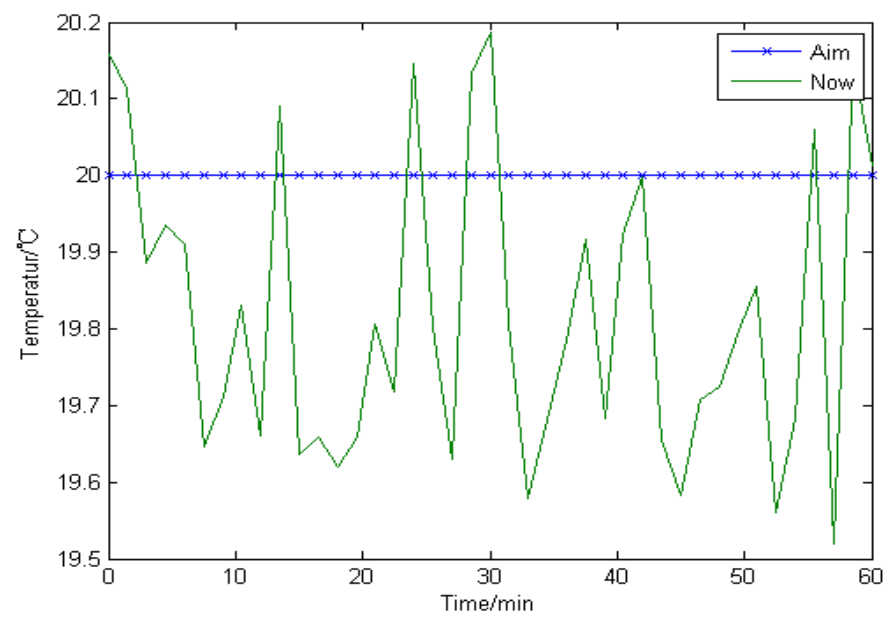

Figure 8. Correlation Curve of Target Temperature and Collection Temperature 


\section{Conclusion}

Proceed from actual engineering, the heating control method of a space camera described in this paper is combined with operation status of software and hardware platform of the camera, it can meet the requirements of operation temperature for space cameras during the working period.

Control method has high stability and veracity. It improves reliability of the space camera.

Control method is simple and explicit, has wide practical application value.

\section{Acknowledgement}

This work was co-supported by the National Natural Science Foundation of China (No. 60506014).

\section{References}

[1] L. H. Chen, Q. W. Wu, Z. T. Luo, et al., "Design for thermal control system of electronic equipment in space camera", Optics and Precision Engineering, vol. 17, no. 9, (2009), pp. 2145-2152.

[2] M. Li, Q. W. Wu and F. Jiang, "Design of thermal control system for three-linear array mapping cameras", Optics and Precision Engineering, vol. 18, no. 6, (2010), pp. 1367-1373.

[3] J. Harvest, S. A. Flescher and R. D. Weintstein, "Modeling of the thermal effects of heat generating devices in close proximity on vertically oriented printed circuit boards for thermal management applications", International Journal of Thermal Sciences, vol. 46, (2007), pp. 253-261.

[4] L. H. Wang, Q. W. Wu, L. Guo, et al., "Thermal Design and Analysis for High Resolution Visible Light Aeronautic Camera", Nfrared and Laser Engineering, vol. 41, no. 5, (2012), pp. 1236-1240.

[5] Z. J. Liu, Y. C. Xiang, D. B. Si, et al., "Design and verification of thermal control system for Chang'E-3 probe", Sci Sin Tech, vol. 44, no. 4, (2014), pp. 353-360.

[6] E. Preter, "The Lunar Base Handbook", New York, The McGraw-Hill Companies, Inc., (1999).

[7] R. L. Chen, Z. Ma, W. G. Yang, et al., "Thermal optical analysis and tests for high resolution space camera", Acta Photonica Sinica, vol. 39, no. 1, (2010), pp. 2068-2071.

[8] R. Li and S. Wang, "Thermal analysis of the baffle structure of the solar space telescope", Science China: Physics, Mechanics and Astronomy, vol.53, no. 9, (2010), pp. 1755-1764.

[9] M. Postman, T. Browen, K. Sembach, et al., "Advanced technology large-aperture space telescope: science drivers and technology developments", Optical Engineering, vol. 51, no. 1, (2012).

[10] R. Li and H. L. Shi, "Thermal effect analysis of main structure in space solar telescope", Nfrared and Laser Engineering, vol. 40, no. 12, (2011), pp. 2425-2431.

[11] Y. Y. Deng and H. Q. Zhang, "Progress in space solar telescope", Science in China Series: Physics, Mechanics and Astronomy, vol. 52, no. 11, (2009), pp. 1655-1659.

[12] G. Wang, "KS-146 Long Focus Aerial Camera", Beijing, PLA Air Command Intelligence Department, (1989).

[13] G. R. Min, “Thermal Control Technology of Satellite”, Beijing, China Astronautics Press, (2009).

[14] J. P. Holman, "Heat Transfer", Beijing, China Machine Press, (2008).

[15] F. P. Incropera, D. P. DeWitt, T. L. Bergman, et al., "Fundamentals of Heat and Mass Transfer", Beijing, Chemistry Industry Press, (2009).

[16] L. Zhang and R. M. Chen, " $\mathrm{TiO}_{2}$-Siloxane Thermal Control Coatings for Protection of Spacecraft Polymers", Chinese Journal of Aeronautics, vol. 17, no. 1, (2004), pp. 53-59.

[17] H. B. Lu and W. Q. Liu, "Investigation of thermal protection system by forward-facing cavity and opposing jet combinatorial configuration", Chinese Journal of Aeronautics, vol. 26, no. 2, (2013), pp. 287-293.

[18] Z. R. Wang, J. Yang, M. Y. Yang, et al., "Investigation on Thermal Contact Conductance Based on Data Analysis Method of Reliability", Chinese Journal of Aeronautics, vol. 25, no. 5, (2012), pp. 791-795.

[19] Z. R. Wang, W. F. Zhang, Q. Y. Tang, et al., "Progress in thermal contact resistance", Rare Metal Materials and Engineering, vol. 40, no. S3, (2011), pp. 211-215. 


\begin{abstract}
Authors
Qing-Lei Zhao, was born in Daqing, China, in 1982. He received the B.S. degree from Tianjin University, Tianjin, China in 2005, and the M.S. degrees from University of Science and Technology of China, Hefei, China in 2010. He is currently working toward the Ph.D. degree in University of Chinese Academy of Sciences, Beijing, China. His area of research includes control of space camera and relative motion dynamics and control.

Ke-Hui Long, was born in Changchun, China, in 1958. She received the B.S. degree from Changchun University of Science and Technology, Changchun, China in 1982. She is currently a Professor and Ph.D. supervisor in University of Chinese Academy of Sciences, China. Her research interests are photoelectric sensor information and remote sensing.

Feng-Wei Guan, was born in Tongliao, China, in 1984. He received the B.S., M.S. degrees from Beijing University of Aeronautics and Astronautics, Beijing, China in 2007 and 2010. His recent interests focus on thermal analysis and space environment simulation.

Liang Huang, was born in Guigang, China, in 1986. He received the B.S. degree from University of Science and Technology of China, Hefei, China in 2008 and the M.S. degrees from University of Chinese Academy of Sciences, Beijing China in 2011. His recent interests focus on FPGA design and space camera control.
\end{abstract}


International Journal of Signal Processing, Image Processing and Pattern Recognition Vol. 8, No. 4 (2015) 